\title{
Asynchronous Computational VSS with Reduced Communication Complexity
}

\author{
Michael Backes \\ Saarland University \& MPI-SWS \\ Germany \\ backes@mpi-sws .org
}

\author{
Amit Datta \\ Carnegie Mellon University \\ U.S.A. \\ amitdatta@cmu.edu
}

\author{
Aniket Kate \\ MMCI, Saarland University \\ Germany \\ aniket@mmci.uni-saarland.de
}

\begin{abstract}
Verifiable secret sharing (VSS) is a vital primitive in secure distributed computing. It allows an untrusted dealer to verifiably share a secret among $n$ parties in the presence of an adversary controlling at most $t$ of them. VSS in the synchronous communication model has received tremendous attention in the cryptographic research community. Nevertheless, recent interest in deploying secure distributed computing over the Internet requires going beyond the synchronous communication model and thoroughly investigating VSS in the asynchronous communication model.

In this work, we consider the communication complexity of asynchronous VSS in the computational setting for the optimal resilience of $n=3 t+1$. The best known asynchronous VSS protocol by Cachin et al. has $O\left(n^{2}\right)$ message complexity and $O\left(\kappa n^{3}\right)$ communication complexity, where $\kappa$ is a security parameter corresponding to the size of the secret. We close the linear complexity gap between these two measures for asynchronous VSS by presenting two protocols with $O\left(n^{2}\right)$ message complexity and $O\left(\kappa n^{2}\right)$ communication complexity. Our first protocol satisfies the standard VSS definition, and can be used in stand-alone VSS scenarios as well as in applications such as Byzantine agreement. Our second and more intricate protocol satisfies a stronger VSS definition, and is useful in all VSS applications including multiparty computation and threshold cryptography.
\end{abstract}

Keywords: Verifiable Secret Sharing, Asynchronous Communication Model, Communication Complexity, Threshold Cryptography, Polynomial Commitments

\section{Introduction}

The notion of secret sharing was introduced independently by Shamir [31] and Blakley [7] in 1979. For integers $n$ and $t$ such that $n>t \geq 0$, an $(n, t)$-secret sharing scheme is a method used by a dealer to share a secret $s$ among a set of $n$ parties in such a way that any subset of $t+1$ or more parties can compute the secret $s$, but subsets of size $t$ or fewer cannot.

In many applications of secret sharing, parties may need to verify the correctness of the values dealt in order to prevent malicious behavior by the dealer. To satisfy this requirement, Chor et al. [17] introduced the concept of verifiable secret sharing (VSS). With its applicability to Byzantine agreement, multiparty computation (MPC) and threshold cryptography, VSS has remained an important area of cryptographic research for the last two decades [12, 14, 18, 20, 21, 28, 29].

Although the literature for VSS is vast, the notion of VSS in the asynchronous communication setting (no bounds on message transfer delays) has not yet received the deserved attention in terms of practical efficiency or theoretical lower bounds. Asynchronous VSS schemes with unconditional 
security have been developed $[1,6,15,27]$; however, these schemes are prohibitively expensive for any realistic use as they need $\Omega\left(\kappa n^{5}\right)$ bits of communication for $\kappa$-bit secrets. In the computational security setting, Cachin et al. [12], Zhou et al. [32], and recently Schultz et al. [30] suggested more practical asynchronous VSS schemes: asynchronous verifiable secret sharing (AVSS), asynchronous proactive secret sharing (APSS) and mobile proactive secret sharing (MPSS), respectively. Of these, AVSS [12] is the most generic and practical asynchronous VSS scheme and it forms the basis for many practical threshold cryptographic protocols such as [22]. AVSS assimilates a bivariate polynomial into Bracha's deterministic reliable broadcast protocol [11], which results into its $O\left(n^{2}\right)$ message complexity (number of messages transferred) and $O\left(\kappa n^{4}\right)$ communication complexity (number of bits transferred) for the optimal resiliency condition of $n=3 t+1$. Cachin et al. [12] further refined the AVSS protocol to reduce the communication complexity to $O\left(\kappa n^{3}\right)$. Nevertheless, a further reduction in the communication complexity is not possible using similar techniques, and a linear complexity gap between the message complexity and the communication complexity still remains.

In this work, we bridge this gap. We present two efficient asynchronous VSS schemes (eAVSS and eAVSS-SC) with different properties (and correspondingly different utilities) with $O\left(n^{2}\right)$ message complexity and $O\left(\kappa n^{2}\right)$ communication complexity.

\section{$1.1 \quad$ Our Contributions}

Kate, Zaverucha and Goldberg [23] define the concept of commitments to polynomials, and devise two schemes PolyCommit ${ }_{\text {DLog }}$ and PolyCommit ${ }_{\text {Ped }}$ that commit to a univariate polynomial of degree $t$ (or less) using a single element of size $O(\kappa)$. Their schemes work in the bilinear pairing setting under the $t$-strong Diffie-Hellman $\left(t\right.$-SDH) assumption [9]. We use their PolyCommit Ped $_{\text {scheme and }}$ a collision-resistant hash function to achieve our goal of asynchronous VSS with $O\left(\kappa n^{2}\right)$ commu-

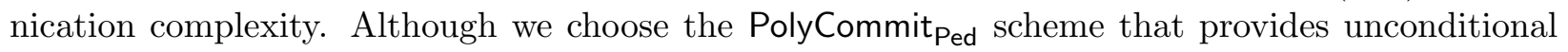
hiding (secrecy) instead of the simpler PolyCommit ${ }_{\text {DLog }}$ scheme that provides computational hiding against the discrete logarithm (DLog) assumption, our protocols work with the PolyCommit ${ }_{\mathrm{DLog}}$ scheme with no modification.

Nevertheless, our schemes are not a straightforward adaptation of the PolyCommit schemes to the bivariate polynomial-based AVSS scheme [12], and not surprisingly, Kate et al. [23] left the applicability of PolyCommit to asynchronous VSS as an open problem. The reason for that, as we elaborate in Section 2.4, is that modifying the PolyCommit schemes to a scheme providing constant-size commitments to bivariate-polynomials used in asynchronous VSS seems difficult if not impossible.

We achieve our goal by taking an entirely different path, bypassing the open problem of obtaining constant-size commitments to bivariate polynomials. We realize asynchronous VSS in two steps: We first present a univariate polynomial-based asynchronous VSS scheme (eAVSS), which guarantees that at least $t+1$ honest parties receive proper shares of the secret, while the remaining honest parties are assured that at least $t+1$ honest parties have received correct shares and can reconstruct the shared secret. This construction is sufficient for stand-alone VSS and for applications such as asynchronous Byzantine agreement (ABA). For applications such as MPC and threshold cryptographic constructions, we then design an efficient stronger asynchronous VSS scheme (eAVSS-SC), which guarantees that every honest party receives its share during the sharing phase. In principle, this is possible by running $n+1$ instances of eAVSS; however, it asks for a broadcast of commitment vectors of size $O(\kappa n)$ which increases the communication complexity to $O\left(\kappa n^{3}\right)$. In eAVSS-SC, we overcome this barrier by aptly modifying the AVSS protocol flow and by hashing the commitments in the vector using a collision-resistant hash function and running a PolyCommit instance over the 
hashed values.

Our schemes have direct implications to the efficiency of all asynchronous VSS applications. Most prominently, using our eAVSS protocol in the modular ABA construction by Canetti and Rabin [13,15], it is possible to obtain the first $O\left(\kappa n^{3}\right)$ communication complexity ABA protocol, which is secure against the adaptive adversary in the standard model.

Organization. In Section 2, we describe our system model and provide a brief overview of the concepts of VSS, polynomial commitments and asynchronous VSS. In Section 3, we define and prove our basic asynchronous VSS protocol (eAVSS), while in Section 4, we define our main asynchronous VSS protocol (eAVSS-SC). In Section 5, we discuss a few interesting applications. An in-depth discussion of the PolyCommit ${ }_{\text {Ped }}$ scheme and corresponding computational assumptions have been added in Appendix A. Due to space restrictions, our proof for eAVSS-SC has been shifted to Appendix B.

\section{Preliminaries}

Our schemes work in the computational security setting. The adversary $\mathcal{A}$ is a probabilistic polynomial time (PPT) algorithm with respect to a security parameter $\kappa$ unless stated otherwise. A function $\epsilon(\cdot): \mathbb{N} \rightarrow \mathbb{R}^{+}$is called negligible if for all $c>0$ there exists a $\kappa_{0}$ such that $\epsilon(\kappa)<1 / \kappa^{c}$ for all $\kappa>\kappa_{0}$. Throughout the rest of this paper, $\epsilon(\cdot)$ denotes a negligible function.

We assume that the shared secret $s$ lies over a finite field $\mathbb{F}_{p}$, where $p$ is a $\kappa$-bit long prime. We use Shamir's polynomial-based secret sharing approach [31], where our polynomials belong to $\mathbb{F}_{p}[x]$ or $\mathbb{F}_{p}[x, y]$.

\subsection{Asynchronous System Model}

Following the adversary and communication model of AVSS given by Cachin et al. [12], we assume an asynchronous fully-connected network of $n$ parties $\mathcal{P}=\left\{P_{1}, P_{2}, \ldots, P_{n}\right\}$, where every pair of parties is connected by an authenticated and private communication link. A special party $P_{d} \in \mathcal{P}$ works as a dealer. The indices for the parties are chosen from $\mathbb{F}_{p}$. Without loss of generality, we assume these indices to be $\{1, \ldots, n\}$.

The adversary $\mathcal{A}$ is $t$-bounded and it can coordinate the actions of up to $t$ out of $n$ parties. The adversary $\mathcal{A}$ is further assumed to be adaptive, and may corrupt a party of its choice at any instance during a protocol execution as long as its total number of corruptions is bounded by $t$. A party is said to be honest if the adversary has not corrupted it. In our asynchronous setting, the adversary $\mathcal{A}$ controls the network and may delay messages between any two honest parties.

However, it cannot read or modify these messages, and it also has to eventually deliver all the messages by honest parties.

\subsection{Verifiable Secret Sharing-VSS}

In many secret sharing applications, a dealer may behave maliciously. This led to the conception of VSS [17].

Definition 2.1. An $(n, t)$-VSS scheme among $n$ parties in $\mathcal{P}=\left\{P_{1}, P_{2}, \ldots, P_{n}\right\}$ with a distinguished party $P_{d} \in \mathcal{P}$ consists of two phases: the sharing (Sh) phase and the reconstruction (Rec) phase. 
Sh phase. A dealer $P_{d}$ distributes a secret $s \in \mathbb{F}_{p}$ among parties in $\mathcal{P}$. At the end of the Sh phase, each honest party $P_{i}$ holds a share $s_{i}$ of the distributed secret $s$.

Rec phase. In this phase, each party $P_{i}$ sends its secret share $s_{i}^{\prime}$ to every party in $\mathcal{P}$ and a reconstruction function is applied in order to compute the secret $s=\operatorname{Rec}\left(s_{1}^{\prime}, s_{2}^{\prime}, \ldots, s_{n}^{\prime}\right)$ or output $\perp$ indicating that $P_{d}$ is malicious. For honest parties $s_{i}^{\prime}=s_{i}$, while for malicious parties $s_{i}^{\prime}$ may be different from $s_{i}$ or even absent.

An $(n, t)$-VSS scheme has the following security requirements:

Secrecy. If the dealer is honest, the adversary who can compromise $t$ parties does not have any more information about $s$ except what is implied by the public parameters.

Correctness. If $P_{d}$ is honest, the reconstructed value should be equal to the dealer's secret s.

Commitment. Even if $P_{d}$ is dishonest, there exists a value $s^{*} \in \mathbb{F}_{p} \cup\{\perp\}$ at the end of the Sh phase, such that all honest parties output $s^{*}$ at the end of the Rec phase.

In this paper, we consider VSS schemes where any malicious behaviour by $P_{d}$ can be identified by the honest parties in the Sh phase itself and the commitment property simplifies to the following: the reconstructed value $z$ should be equal to a shared secret $s \in \mathbb{F}_{p}$ that gets fixed at the end of the Sh phase.

Many VSS applications (e.g., threshold cryptography and MPC) avoid participation by all parties once the Sh phase is over. It is required that messages from any $t+1$ honest parties (or any $2 t+1$ parties) are sufficient to reconstruct the shared secret $s$. For these applications, we require a stronger commitment property that we refer as the strong commitment requirement.

Strong Commitment. Even if $P_{d}$ is dishonest, there exists a value $s^{*} \in \mathbb{F}_{p}$ at the end of the Sh phase, such that $s^{*}$ is reconstructed regardless of the subset of parties (of size greater than 2t) chosen by the adversary in the Rec phase.

Further, some VSS schemes achieve a weaker (computational) secrecy guarantee.

Weak Secrecy. A t-limited adversary who can compromise t parties cannot compute $s$ during the Sh phase.

We also give the following definitions for the complexity measures.

Definition 2.2 (Message Complexity). The message complexity is defined as the total number of messages exchanged between the parties participating in a scheme.

Definition 2.3 (Communication Complexity). The communication complexity is defined as the total number of bits exchanged between the parties taking into consideration every message that has been transmitted.

A variant of VSS considers dealer $P_{d}$ to be an external party (i.e., $P_{d} \notin \mathcal{P}$ ) and allows the adversary to corrupt $P_{d}$ and up to $t$ additional parties in $\mathcal{P}$. All our protocols also work in this stronger setting.

Assuming a broadcast channel, Feldman [20] developed the first non-interactive and efficient VSS scheme and Pedersen [28,29] presented a modification to it. Both protocols obtain the strong commitment property. In terms of secrecy, Feldman VSS achieves the weak secrecy property, while Pedersen VSS achieves the stronger form.

\subsection{Use of Commitments in VSS}

A verification mechanism for a consistent dealing is fundamental to VSS. It is achieved using distributed computing techniques in the information-theoretic security setting. In the computational setting that we focus in this paper, the commitment schemes provide an efficient alternative. 
A commitment scheme allows a committer to publish a value, called the commitment (say $\mathcal{C}$ ), which binds her to a message $s$ (binding) without revealing it (hiding). Later, she may open the commitment $\mathcal{C}$ and reveal the committed message $m$ to a verifier, who can check that the message is consistent with the commitment. In particular, the computational VSS schemes utilize the commitments to the shared polynomials. Kate et al. [23] formalize this concept of polynomial commitments. Here, we present a refined version of their polynomial commitment (PolyCommit) definition for polynomial of degree $\leq t$.

Definition 2.4. A PolyCommit scheme consists of the following algorithms:

$\operatorname{Setup}\left(1^{\kappa}, t\right)$ generates system parameters $S P$ to commit to a polynomial of degree $\leq t$. In these system parameters, let $\mathbb{G}$ be an algebraic structure for commitments. Setup is run by a trusted or distributed authority. SP can also be standardized for repeated use.

Commit $(\mathbf{S P}, \phi(x))$ outputs a commitment $\mathcal{C}$ to a polynomial $\phi(x)$ for the system parameters $S P$, and some associated decommitment information d. (In some constructions, $d$ can be null.)

Open $(\mathbf{S P}, \mathcal{C}, \phi(x),[d])$ outputs the polynomial $\phi(x)$ used while creating the commitment, with decommitment information $d$.

VerifyPoly $(\mathbf{S P}, \mathcal{C}, \phi(x),[d])$ verifies that $\mathcal{C}$ is a commitment to $\phi(x)$, created with decommitment information d. If so, the algorithm outputs 1 , otherwise it outputs 0.

CreateWitness $(\mathbf{S P}, \phi(x), i,[d])$ outputs $\left\langle i, \phi(i), w_{i}, d_{i}\right\rangle$, where $w_{i}$ is a witness and $d_{i}$ is the decommitment information for the evaluation $\phi(i)$ of $\phi(x)$ at the index $i$. This algorithm is optional.

$\operatorname{Verify} \operatorname{Eval}\left(\mathbf{S P}, \mathcal{C}, i, \phi(i),\left[d_{i}, w_{i}\right]\right)$ verifies that $\phi(i)$ is indeed the evaluation at the index $i$ of the polynomial committed in $\mathcal{C}$. If so, the algorithm outputs 1 , otherwise it outputs 0.

Given SP $\leftarrow \operatorname{Setup}\left(1^{\kappa}, t\right)$, a PolyCommit scheme satisfies the following properties:

Correctness. Let $\mathcal{C} \leftarrow$ Commit $(S P, \phi(x))$. For a commitment $\mathcal{C}$ generated by Commit(SP, $\phi(x))$, and all $\phi(x) \in \mathbb{Z}_{p}[x]$, any $\left\langle i, \phi(i), w_{i}, d_{i}\right\rangle$ output by CreateWitness $(S P, \phi(x), i)$ is successfully verified by VerifyEval(SP, $\left.\mathcal{C}, i, \phi(i), d_{i}, w_{i}\right)$.

Strong Correctness. For all adversaries $\mathcal{A}, \operatorname{Pr}\{(\mathcal{C},\langle\phi(x), d\rangle) \leftarrow \mathcal{A}(S P): \operatorname{deg}(\phi(x))>t\}=$ $\epsilon(\kappa)$.

Polynomial Binding. For all adversaries $\mathcal{A}$ :

$$
\operatorname{Pr}\left\{\left(\mathcal{C},\langle\phi(x), d\rangle,\left\langle\phi^{\prime}(x), d^{\prime}\right\rangle\right) \leftarrow \mathcal{A}(S P):\left(\begin{array}{c}
\operatorname{VerifyPoly}(S P, \mathcal{C}, \phi(x), d)=1 \\
\operatorname{VerifyPoly}\left(S P, \mathcal{C}, \phi^{\prime}(x), d^{\prime}\right)=1 \\
\wedge \phi(x) \neq \phi^{\prime}(x)
\end{array}\right\}=\epsilon(\kappa) .\right.
$$

Evaluation Binding. For all adversaries $\mathcal{A}$ :

$\operatorname{Pr}\left\{\left(\mathcal{C},\left\langle i, \phi(i), d_{i}, w_{i}\right\rangle,\left\langle i, \phi(i)^{\prime}, d_{i}^{\prime}, w_{i}^{\prime}\right\rangle\right) \leftarrow \mathcal{A}(S P):\left(\begin{array}{c}\operatorname{VerifyEval}\left(S P, \mathcal{C}, i, \phi(i), d_{i}, w_{i}\right)=1 \\ \operatorname{VerifyEval}\left(S P, \mathcal{C}, i, \phi(i)^{\prime}, d_{i}^{\prime}, w_{i}^{\prime}\right)=1 \\ \wedge \phi(i) \neq \phi(i)^{\prime}\end{array}\right)=\epsilon(\kappa)\right.$.

(Unconditional) Hiding. Given $\langle S P, \mathcal{C}\rangle$ and $\left\{\left\langle i_{j}, \phi\left(i_{j}\right), d_{i_{j}}, w_{\phi_{i_{j}}}\right\rangle: j \in[1, \operatorname{deg}(\phi)]\right\}$ for some $\phi(x) \in_{R} \mathbb{Z}_{p}[x]$ such that VerifyEval $\left(S P, \mathcal{C}, i_{j}, \phi\left(i_{j}\right), d_{i_{j}}, w_{\phi_{i_{j}}}\right)=1$ for each $j$, a computationally unbounded adversary $\hat{\mathcal{A}}$ has no information about $\phi(\hat{j})$ for any unqueried index $\hat{j}$.

The above strong correctness property is not present in the original PolyCommit definition. We include it as restricting degree of the committed polynomial by a threshold $t$ is required for VSS. Further, a weaker form of hiding is also possible, where a computationally bounded adversary $\mathcal{A}$ cannot compute $\phi(\hat{j})$ for any unqueried index $\hat{j}$. We consider the unconditional hiding property in the paper. 
In literature, VSS protocols utilized commitments to the coefficients or evaluations of shared polynomials as polynomial commitments. They used two commitment schemes. Given $g$ and $h$ as two random generators of a multiplicative group of order $p$, Feldman VSS and its variants use a commitment scheme of the form $g^{s}$ with computational hiding under the discrete logarithm (DLog) assumption and unconditional binding. Pedersen [28] presented another commitment of the form $g^{s} h^{r}$ with unconditional hiding but computational binding under the DLog assumption. The hiding property of the commitment scheme leads to the secrecy property of VSS, while the binding property leads to the correctness property of VSS. Both of these commitment schemes also trivially satisfy the commitment property of VSS by the fact that the size of a commitment to a polynomial $\phi(x) \in \mathbb{Z}_{p}[x]$ is equal to $\operatorname{deg}(\phi)+1$. In the complexity terms, the size of commitment is $O(n)$ (since for optimal resiliency, $\operatorname{deg}(\phi)=t=\left\lfloor\frac{n-1}{2}\right\rfloor$ ). However, the commitment to a shared polynomial has to be broadcast to all parties, which results in a linear-size broadcast for Feldman VSS, and a linear complexity gap between the message and the communication complexities.

Kate et al. [23] close this gap for Feldman VSS and its variants using a commitment that commits to the entire univariate polynomial using a single element. In particular, they define two polynomial commitment (PolyCommit) schemes: PolyCommit ${ }_{\text {DLog }}$ and PolyCommit Ped $_{\text {, both of which }}$ works in the bilinear pairing setting with $\Theta(t)$ system parameters. PolyCommit ${ }_{\text {DLog }}$ attains hiding under the DLog assumption, binding under the $t$-strong Diffie-Hellman ( $t$-SDH) assumption [9], and strong correctness under the $t$-polynomial Diffie-Hellman assumption (refer to Appendix A.1 for the definitions of assumptions). Using a technique similar to Pedersen commitments, they also define PolyCommit $_{\text {Ped }}$, which attains unconditional hiding and computational binding under the $t$-SDH assumption. These constructions are based on an algebraic property of polynomials $\phi(x) \in \mathbb{F}_{p}[x]$ that $(x-i)$ perfectly divides the polynomial $\phi(x)-\phi(i)$ for any $i \in \mathbb{F}_{p}$.

In this work, we extend the utility of the PolyCommit concept to asynchronous VSS. We choose

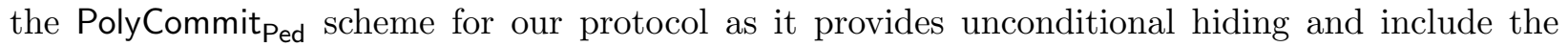
PolyCommit ${ }_{\text {Ped }}$ construction in Appendix A.

\subsection{Asynchronous VSS}

The asynchronous communication setting places no bounds on message delays. Consequently, there is no trivially available broadcast channel, and Feldman VSS and its variants do not guarantee a correct completion. This gives rise to the concept of asynchronous VSS for optimal resilience of $n=3 t+1$.

An asynchronous VSS protocol requires the liveness and agreement properties along with the secrecy, correctness and commitment properties defined in Section 2.2

Definition 2.5. An asynchronous VSS protocol having $n \geq 3 t+1$ parties with a $t$-limited Byzantine adversary satisfies the following conditions:

Liveness. If the dealer $P_{d}$ is honest in the Sh phase, then all honest parties complete the Sh phase.

Agreement. If some honest party completes the Sh phase, then all honest parties will eventually complete the Sh phase. If all honest parties subsequently start the Rec phase, then all honest parties will complete the Rec phase.

Correctness, Commitment and Secrecy. as defined in Section 2.2.

For VSS applications such as MPC, we need VSS that has identical secrecy, correctness, liveness and agreement properties as in Definition 2.5, but a stronger commitment property as defined in Section 2.2. In other words, there exists a $t$-degree polynomial $f(x)$ such that a share $s_{i}$ held by every honest party $P_{i}$ at the end of the sharing phase is equal to $f(i)$. 
As discussed in the introduction, three computational VSS schemes have been suggested for the asynchronous setting: AVSS [12], APSS [32], and MPSS [30]. Of these, AVSS [12] provides the first and the most practical asynchronous VSS scheme. In the AVSS methodology, secret sharing is integrated into a reliable broadcast primitive [11]. This results into its $O\left(n^{2}\right)$ messages complexity. Here, the commitments to the secret and its shares are broadcast, and the shares themselves are appropriately appended to the broadcast commitments so that parties receive their shares while maintaining their secrecy. To overcome an adversarial dealer that does not provide some honest party with its correct share, parties send sub-shares to each other along with the broadcasted commitment. The victim party then computes its share from the received sub-shares. AVSS implements this using bivariate polynomial-based secret sharing, which leads to a commitment (or broadcast) of size $\Theta\left(\kappa n^{2}\right)$ and correspondingly $O\left(\kappa n^{4}\right)$ bits of communication. In the same paper, Cachin et al. improve their AVSS scheme by reducing the commitment-size to $\Theta(\kappa n)$, which results in $O\left(\kappa n^{3}\right)$ bits of communication. A linear gap between the message complexity and the communication complexity still remains.

A Mismatch between AVSS and PolyCommit. It is tempting to consider filling this gap for AVSS using a bivariate PolyCommit scheme that commits to an entire bivariate polynomial using a constant-size commitment; however, this does not seem to be possible with the existing PolyCommit methodology. PolyCommit schemes use the algebraic property that, for $\phi(x) \in \mathbb{F}_{p}[x]$, $(x-i)$ perfectly divides the polynomial $\phi(x)-\phi(i)$ for any $i \in \mathbb{F}_{p}$. However, such a perfect and direct relation is not known between a bivariate polynomial $\phi(x, y)$ and its evaluations $\phi(i, j)$ for any $i, j \in \mathbb{F}_{p} \cdot{ }^{1}$ Therefore, we will have to use two-stage properties involving univariate polynomials (e.g., $(x-i)(y-j)$ perfectly divides the polynomial $\phi(x, y)-\phi(i, y)-\phi(x, j)+\phi(i, j)$ for any $\left.i, j \in \mathbb{F}_{p}\right)$. However, this does not work either because even though the $t$-SDH problem to find $\left\langle c, g^{\frac{1}{\alpha+c}}\right\rangle$ for any value of $c \in \mathbb{Z}_{p}$ given $\left\langle g, g^{\alpha}, g^{\alpha^{2}}, \ldots, g^{\alpha^{t}}\right\rangle$ is conjectured to be hard, its exponential version to find a pair $\left\langle g^{c}, g^{\frac{1}{\alpha+c}}\right\rangle$ is easy.

A closer look at AVSS reveals that further reducing the commitment-size in the hash-based approach of Cachin et al. using a univariate PolyCommit scheme also does not work: Cachin et al. hash the shares (or the univariate polynomials) for $n$ parties and the secret. These $n+1$ hashed values sent to each party constitute a polynomial of degree $n$ instead of degree $t$ of the underlying bivariate polynomial. This requires an honest party to wait for (constant-size) messages from all $n$ parties in AVSS, which is impossible in the asynchronous setting.

As a result, we have to work towards our goal of asynchronous VSS with $O\left(\kappa n^{2}\right)$ in a different way. In the next section, we provide an asynchronous VSS that satisfies the basic VSS definition, and extend it to a stronger version with applicability in all known VSS applications in Section 4.

\section{3 eAVSS: Asynchronous VSS Protocol}

In this section, we present a protocol (eAVSS) with $O\left(n^{2}\right)$ message complexity and $O\left(\kappa n^{2}\right)$ communication complexity and that satisfies Definition 2.5 of asynchronous VSS. The eAVSS protocol guarantees that at least $t+1$ honest parties receive proper shares of the secret committed using a $t$-degree univariate polynomial during the Sh phase, while the remaining honest parties are assured that there are at least $t+1$ honest parties that have received correct shares and can complete the Rec phase. The protocol is sufficient for applications such as Byzantine agreement and stand-alone

\footnotetext{
${ }^{1}$ This is equivalent to derivatives in calculus, where complete derivation of a multi-variable equation is not possible and partial derivatives are employed.
} 


\section{Sh Phase}

\section{Dealer $P_{d}$ with the secret $s$}

- Select a polynomial $\phi(x)$ of degree $t$ such that $\phi(0)=s$.

- Compute a commitment $\langle\mathcal{C}, \widehat{\phi}(x)\rangle \leftarrow \operatorname{Commit}(\mathrm{SP}, \phi(x))$ and witnesses $w_{j} \leftarrow$ CreateWitness $(\mathrm{SP}, \phi(x), \widehat{\phi}(x), j)$ for $j \in[1, n]$.

- Send (send, $\left.\mathcal{C}^{\prime}=\mathcal{C}, \phi(j), \widehat{\phi}(j), w_{j}\right)$ to every party $P_{j}$.

\section{Every party $P_{i}$}

- On receiving a message (send, $\left.\mathcal{C}^{\prime}, \phi(i), \widehat{\phi}(i), w_{i}\right)$ from $P_{d}$, run VerifyEval(SP, $\left.\mathcal{C}^{\prime}, i, \phi(i), \widehat{\phi}(i), w_{i}\right)$. If the verification succeeds, set $\mathcal{C}=\mathcal{C}^{\prime}$ and send (echo, $\mathcal{C}^{\prime}$ ) to all parties.

- On receiving (echo, $\left.\mathcal{C}^{\prime}\right)$ messages from $(n-t)$ parties:

- For $\mathcal{C}^{\prime}=\mathcal{C}$, send (ready, share, $\mathcal{C}^{\prime}$ ) to all parties;

- For $\mathcal{C}^{\prime} \neq \mathcal{C}$, discard $\left\langle\phi(j), \widehat{\phi}(j), w_{j}\right\rangle$, set $\mathcal{C}=\mathcal{C}^{\prime}$, and send (ready, no-share, $\mathcal{C}^{\prime}$ ) to all parties.

- If a ready message has not been sent, then on receiving (ready, $*, \mathcal{C}^{\prime}$ ) messages from $t+1$ parties:

- For $\mathcal{C}^{\prime}=\mathcal{C}$, send (ready, share, $\mathcal{C}^{\prime}$ ) to all parties;

- For $\mathcal{C}^{\prime} \neq \mathcal{C}$, discard $\left\langle\phi(j), \widehat{\phi}(j), w_{j}\right\rangle$, set $\mathcal{C}=\mathcal{C}^{\prime}$, and send (ready, no-share, $\mathcal{C}^{\prime}$ ) to all parties.

- On receiving (ready, $*, \mathcal{C}^{\prime}$ ) messages from at least $(n-t)$ parties such that share flags are set in at least $t+1$ of those, complete the Sh phase with commitment $\mathcal{C}=\mathcal{C}^{\prime}$ (and $\left\langle\phi(i), \widehat{\phi}(i), w_{i}\right\rangle$ if present).

\section{Every party $P_{i}$}

\section{Rec Phase}

- Send (rec-share, $\left.\phi(i), \widehat{\phi}(i), w_{i}\right)$ to all parties, if it has sent a (ready, share, $\mathcal{C}$ ) message in the Sh phase.

- On receiving $t+1$ rec-share messages verified using $\operatorname{Verify} \operatorname{Eval}\left(\mathrm{SP}, \mathcal{C}, j, \phi(j), \widehat{\phi}(j), w_{j}\right)$, interpolate the secret as $s=\phi(0)$.

Figure 1: Protocol eAVSS for Asynchronous VSS $(n \geq 3 t+1)$

VSS. The protocol construction is significantly simpler than the AVSS protocol [12] and it has a protocol flow similar to a VSS protocol for non-homomorphic commitments [5].

\subsection{Construction}

We take a PolyCommit ${ }_{\text {Ped }}$ commitment Setup instance SP $\leftarrow \operatorname{Setup}\left(1^{\kappa}, t\right)$. We choose PolyCommit ${ }_{\text {Ped }}$ due to its unconditional hiding property and the constant size of the commitments. It can, however, be replaced by any polynomial commitment scheme.

The dealer $P_{d}$ starts off the protocol by choosing a univariate polynomial $\phi(x)$ with $\phi(0)=s$, and computing a polynomial commitment $\langle\mathcal{C}, d\rangle \leftarrow \operatorname{Commit}(\mathrm{SP}, \phi(x))$ and corresponding witnesses

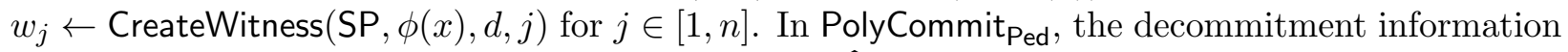
$d$ is a $t$-degree polynomial, which is represented as $\widehat{\phi}(x)$ in the rest of the paper. $P_{d}$ then sends (send, $\left.\mathcal{C}, \phi(j), \widehat{\phi}(j), w_{j}\right)$ messages to all parties and the parties verify their shares against the received commitment $\mathcal{C}$. In the rest of the protocol, the parties try to agree on $\mathcal{C}$. Unlike AVSS, the parties in eAVSS do not exchange their common evaluations of a bivariate polynomial; they only verify consistency of the received shares (if any) with $\mathcal{C}$ locally. If the dealer is dishonest, some honest parties may not receive shares consistent with $\mathcal{C}$; however, they still help to reach an 
agreement on $\mathcal{C}$ once they are assured that at least $t+1$ honest parties have received shares and witnesses consistent with $\mathcal{C}$. We describe the protocol in Figure 1. Note that commitment $\mathcal{C}$ is set to $\perp$ initially. An honest party accepts only one message of a kind from any other party, and without loss of generality, we assume that every party chooses only the first message.

The protocol requires $O\left(n^{2}\right)$ messages as decided by its echo and ready messages. Use of PolyCommit ensures that all messages are of a constant size, and results in $O\left(\kappa n^{2}\right)$ communication complexity.

\subsection{Analysis}

Theorem 3.1. Given a PolyCommit scheme that satisfies Definition 2.4, eAVSS is an asynchronous VSS protocol that satisfies Definition 2.5.

Proof. To prove the theorem, we show that protocol eAVSS satisfies liveness, agreement, correctness, commitment, secrecy properties of asynchronous VSS according to Definition 2.5. Our analysis is based on the properties of the polynomial commitment scheme used.

We start by proving the following two claims.

Claim 3.2. If some honest party has agreed on $\mathcal{C}$, then every honest party will eventually agree on $\mathcal{C}$.

Proof. We first prove by contradiction that if $P_{i}$ be the first honest party to send ready message containing $\mathcal{C}$, then a ready message sent by every other honest party $P_{j}$ will contain $\mathcal{C}$. Assume an honest party $P_{j}$ sends a ready message with $\overline{\mathcal{C}}$ such that $\mathcal{C} \neq \overline{\mathcal{C}}$. Being the first honest party to send a ready message with $\mathcal{C}$ party $P_{i}$ must have received (echo, $\mathcal{C}$ ) from at least $n-t$ parties of which at least $n-2 t$ were honest. $P_{j}$ can send $\overline{\mathcal{C}}$ only after one of the following two events and in both cases we arrive at a contradiction:

1. $P_{j}$ can send (ready, $\left.\overline{\mathcal{C}}\right)$ after receiving $($ echo, $\overline{\mathcal{C}}$ ) from at least $n-t$ parties. As $n \geq 3 t+1$, $(n-t)+(n-t)-n=n-2 t \geq t+1$ parties must have sent echo with both $\mathcal{C}$ and $\overline{\mathcal{C}}$. This implies that at least one honest party sent echo messages of two types, which is impossible.

2. $P_{j}$ can also send $($ ready, $\overline{\mathcal{C}})$ after receiving $n-2 t$ (ready, $\left.*, \overline{\mathcal{C}}\right)$ messages. For $n \geq 3 t+1$, $n-2 t \geq t+1$. Therefore, there is at least one honest party (say $P_{k}$ ), who sent $\overline{\mathcal{C}}$ in its ready message to $P_{j}$. This means that one of the events (1) or (2) must have occurred with the honest party $P_{k}$. If we argue in a recursive manner, we reach some honest party who must have experienced event (1), which is a contradiction.

Therefore, no two honest parties will send ready messages containing different commitments.

A honest party agrees on $\mathcal{C}$ only after receiving at least $n-t$ ready messages such that at least $t+1$ contain share. Therefore, $n-2 t \geq t+1$ honest parties must have sent ready message and at least one honest party must have sent a ready message containing share. ready messages from $t+1$ or more parties will eventually reach all remaining honest parties and they will send ready messages with the same $\mathcal{C}$, as discussed above. As the number of honest parties is at least $n-t$, every honest party will receive at least $n-t$ ready messages.

It, however, remains to show that every honest party will eventually receive at least $t+1$ ready messages with the share flag. From the above paragraph, at least one honest party must have sent a ready message for $\mathcal{C}$ after receiving $n-t$ echo messages for $\mathcal{C}$ and out of those at least $n-2 t \geq t+1$ are sent by honest parties. As an honest party sends an echo message only after receiving a verifiable send message from the dealer, at least $t+1$ honest parties must have received 
their shares from the dealer. As every honest party eventually sends a ready message, these $t+1$ parties will also certainly send ready messages and importantly, they will contain the share flag. Therefore, every honest party will eventually receive $n-t$ ready messages for $\mathcal{C}$ with $t+1$ share flags and agree on $\mathcal{C}$.

Claim 3.3. If some honest party agrees on $\mathcal{C}$, then there exists a subset of at least $n-2 t \geq t+1$ honest parties such that each of those holds an evaluation of a degree-t polynomial consistent with $\mathcal{C}$.

Proof. From the proof of Claim 3.2, $n-2 t$ honest parties will eventually send out ready messages for $\mathcal{C}$ with share; these $n-2 t$ honest parties have received verifiable send messages for $\mathcal{C}$ from the dealer $P_{d}$. Note that these honest parties never update $\mathcal{C}$, and eventually agree on the same $\mathcal{C}$ by Claim 3.2. Due to the strong correctness and polynomial binding properties of PolyCommit Ped, $_{\text {, }}$ there is a unique $t$-degree of polynomial $\phi(x)$ committed by $\mathcal{C}$. Therefore, evaluations available with these $n-2 t \geq t+1$ parties implicitly defines $\phi(x)$ that is consistent with $\mathcal{C}$.

Liveness. If the dealer $P_{d}$ is honest, then every honest party will eventually receive verifiable send message sent by $P_{d}$ and will send an echo message and then a ready message. As there are $n-t \geq 2 t+1$ honest parties, they will finally agree on $\mathcal{C}$ and complete the Sh phase.

Agreement. A party completes its $S$ phase as soon as it agrees on a commitment $\mathcal{C}$. Claim 3.2 suggests that if an honest party agrees on $\mathcal{C}$, then every honest party will eventually agree on $\mathcal{C}$. Therefore, if one honest party completes the Sh phase, then every honest party will complete its Sh phase.

For agreement in the Rec phase, Claim 3.3 shows that there is a subset of at least $t+1$ honest parties each holding an evaluation of a degree- $t$ polynomial $\phi(x)$ that is consistent with $\mathcal{C}$. As every honest party participates in the Rec phase, $t+1$ correct evaluations of $\phi(x)$ associated with $\mathcal{C}$ are available in the Rec phase, and the secret $s=\phi(0)$ can be interpolated by every honest party.

Correctness. Assume that the dealer has shared a secret $s$ using a polynomial $\phi(x)$, and has remained honest throughout the execution of the Sh phase. Let $\mathcal{C}$ be the commitment to $\phi(x)$ sent by the dealer. Given correctness of the polynomial commitment scheme, all honest parties will receive correct shares of the secret $s$ that is consistent with $\mathcal{C}$. Therefore, as we discussed above for agreement, the same secret $s$ will be reconstructed by the parties.

Commitment. We prove the commitment by contradiction. Assume that two different honest parties $P_{i}$ and $P_{j}$ reconstruct different $s^{\prime}$ and $s^{\prime \prime}$ such that $s^{\prime} \neq s^{\prime \prime}$, The maximum possible degree of the committed polynomial is $t$ due to strong correctness of PolyCommit ${ }_{\text {Ped }}$. Therefore, each of them must have agreed upon different commitments (say) $\mathcal{C}^{\prime}$ and $\mathcal{C}^{\prime \prime}$ in the Sh phase. However, this contradicts with Claim 3.2. Therefore, a unique value $s^{*} \in \mathbb{F}_{p}$ will be reconstructed by all honest parties.

Secrecy. We have to show that if the dealer $P_{d}$ is honest, then the adversary $\mathcal{A}$ obtains no information about the secret $s$. A $t$-limited adversary will be able to obtain the $t$ messages of the form (send, $\mathcal{C}, w_{i}, \phi(i)$ ). Due the hiding property for polynomial commitments, given only $t$ such messages it is impossible to reconstruct polynomial $\phi(x)$ (of degree $t$ ) and correspondingly the dealer's secret $s=\phi(0)$. 


\section{4 eAVSS-SC: Asynchronous VSS Protocol with Strong Commit- ment}

Although protocol eAVSS in Section 3 does not attain the strong commitment property, it can be used as a component of a VSS protocol that satisfies the property. The most intuitive way to realize such a VSS scheme is to make $P_{d}$ execute $(n+1)$ correlated instances of eAVSS, where the secret $s$ is shared using the first instance (say) eAVSS $_{0}$ and the associated shares or polynomial evaluations for all $n$ parties in eAVSS ${ }_{0}$ are themselves shared using $n$ instances eAVSS ${ }_{j}$ for $j \in[1, n]$. Once all eAVSS $_{j}$ instances complete their Sh phases, a subset of $t+1$ or more honest parties provide every $P_{j}$ its share in eAVSS 0 by running the Rec phase of eAVSS ${ }_{j}$, and by sending their verifiable shares of eAVSS ${ }_{j}$ to only $P_{j}$. It is possible to combine send, echo and ready messages for all $n+1$ instances to keep the message complexity the same as that of AVSS and eAVSS, i.e., $O\left(n^{2}\right)$. However, to broadcast all associated commitments, the communication complexity becomes $O\left(\kappa n^{3}\right)$, which is no better than that of AVSS [12]. In protocol eAVSS-SC, we overcome this drawback using a collision-resistant hash function.

\subsection{Construction}

Here, the dealer $P_{d}$ shares the secret $s$ using a symmetric bivariate polynomial $\phi(x, y)$ such that $\phi(0,0)=s$. The dealer commits to this bivariate polynomial using the univariate PolyCommit scheme twice. In Section 2.4, we observed that constant-size commitments to bivariate polynomials seem difficult, if not impossible. Here, we overcome this hurdle using PolyCommit over the hashed univariate PolyCommit values. ${ }^{2}$ We provide an expository description of protocol eAVSS-SC in Figure 2. Notice that although we use send, echo, and ready messages similar to AVSS, our message structures and their utilities are significantly different from those of AVSS. These message structures

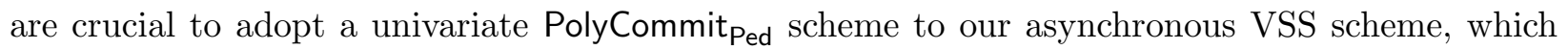
uses bivariate polynomials.

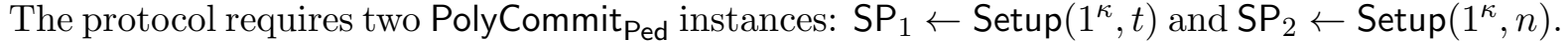
$P_{d}$ runs $n+1$ eAVSS instances with polynomials $\phi(x, 0), \phi(x, 1), \ldots, \phi(x, n)$. Let $\mathcal{C}_{0}, \mathcal{C}_{1}, \ldots, \mathcal{C}_{n}$ be the commitments for these $n+1$ instances. $P_{d}$ also computes an $n$-degree polynomial $h_{\mathcal{C}}(x)$ from $H\left(\mathcal{C}_{0}\right), H\left(\mathcal{C}_{1}\right), \ldots, H\left(\mathcal{C}_{n}\right)$, where $H: \mathbb{G} \rightarrow \mathcal{F}_{p}$ is a collision-resistant hash function and broadcasts a commitment $\zeta$ to $h_{\mathcal{C}}(x)$. This makes the size of the commitment in this scheme a constant-sized one. The dealer cannot cheat with $\phi(x, y)$ as the PolyCommit ${ }_{\text {Ped }}$ scheme is binding and the hash function is collision-resistant. When all honest parties agree on $\zeta$, they implicitly agree on $\mathcal{C}_{0}, \mathcal{C}_{1}, \ldots, \mathcal{C}_{n}$. As $t+1$ or more honest parties have received all required shares $\phi_{i}(x)=\phi(x, i)$ and $\hat{\phi}_{i}(x)=\hat{\phi}(x, i)$, and commitments $\overrightarrow{\mathcal{C}}$, they can provide all parties their required shares, commitments and witnesses

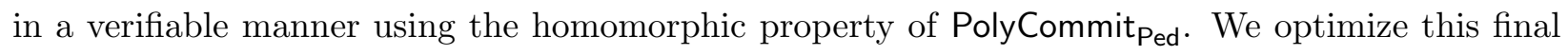
step by attaching the required shares, witnesses and commitments to the ready messages.

From the protocol description, it is apparent that the message complexity is $O\left(n^{2}\right)$. As we use the PolyCommit ${ }_{\text {Ped }}$ scheme that commits to univariate polynomials using a single element, the communication complexity is $O\left(\kappa n^{2}\right)$. Note that although the size of send messages is $O(\kappa n)$, only $n$ such messages are delivered; thus, the communication complexity does not exceed $O\left(\kappa n^{2}\right)$.

For simplicity of the description, we define our protocol with a symmetric bivariate polynomial. It is easily possible to avoid this symmetry requirement in the protocol without any asymptotic increase in the complexity measures.

\footnotetext{
${ }^{2}$ Note that our scheme is not a generic constant-size commitment scheme for bivariate polynomials and some care has to be taken before applying it in other applications; e.g., our scheme cannot be applied to the main as well as the refined AVSS protocols [12] without making their computational complexity exponential $O\left(\begin{array}{l}n \\ t\end{array}\right)$.
} 


\section{Dealer $P_{d}$ with the secret $s$}

\section{Sh Phase}

- Choose a symmetric $t$-degree bivariate polynomial $\phi(x, y)$ such that $\phi(0,0)=s$ and $\phi(i, j)=\phi(j, i)$.

- Commit to $\phi(x, y)$ using a vector $\overrightarrow{\mathcal{C}}=\left\{\mathcal{C}_{j}\right\}_{j \in[0, n]}$, where $\left\langle\mathcal{C}_{j}, \hat{\phi}_{j}(x)\right\rangle \leftarrow \operatorname{Commit}\left(\mathrm{SP}_{1}, \phi_{j}(x)\right), \phi_{j}(x)=$ $\phi(x, j)$ and $\hat{\phi}(x, y)$ is symmetric. Also, compute witness vectors $\overrightarrow{\mathcal{W}}_{j}=\left\{w_{j}^{k}\right\}_{k \in[0, n]}$ for every party $P_{j}$ such that $w_{j}^{k} \leftarrow$ CreateWitness $\left(\mathrm{SP}_{1}, \phi_{k}(x), \hat{\phi}_{k}(x), j\right)$.

- Compute an $n$-degree polynomial $h_{\mathcal{C}}(x)$ from $H\left(\mathcal{C}_{0}\right), H\left(\mathcal{C}_{1}\right), \ldots, H\left(\mathcal{C}_{n}\right)$, where $H: G \rightarrow \mathcal{F}_{p}$ is a collision-resistant hash function and commit to it $\left\langle\zeta, \widehat{h}_{\mathcal{C}}(x)\right\rangle \leftarrow \operatorname{Commit}\left(\mathrm{SP}_{2}, h_{\mathcal{C}}(x)\right)$

- Send (send, $\left.\zeta^{\prime}=\zeta, \overrightarrow{\mathcal{C}}^{\prime}=\overrightarrow{\mathcal{C}}, \widehat{h}_{\mathcal{C}}(x), \overrightarrow{\mathcal{W}}_{j}, \phi_{j}(x), \hat{\phi}_{j}(x)\right)$ to every party $P_{j}$.

Every party $P_{i}$

- On receiving a message (send, $\left.\zeta^{\prime}, \overrightarrow{\mathcal{C}}^{\prime}, \widehat{h}_{\mathcal{C}}(x), \overrightarrow{\mathcal{W}}_{i}, \phi_{i}(x), \hat{\phi}_{i}(x)\right)$ from $P_{d}$, verify its correctness:

- interpolate the complete $\overrightarrow{\mathcal{C}^{\prime}}$ from any of its $t+1$ elements to assert the degree $t$ of the polynomial;

- compute $h_{\mathcal{C}}(x)$ from $\overrightarrow{\mathcal{C}^{\prime}}$ and VerifyPoly $\left(\mathrm{SP}_{2}, \zeta^{\prime}, h_{\mathcal{C}}(x), \widehat{h}_{\mathcal{C}}(x)\right)$

- VerifyPoly $\left(\mathrm{SP}_{1}, \mathcal{C}_{i}^{\prime}, \phi_{i}(x), \hat{\phi}_{i}(x)\right)$

- VerifyEval $\left(\mathrm{SP}_{1}, \mathcal{C}_{j}^{\prime}, i, \phi_{j}(i)\left[=\phi_{i}(j)\right], \hat{\phi}_{j}(i)\left[=\hat{\phi}_{i}(j)\right], w_{i}^{j}\right)$ for every $j \in[0, n]$.

Upon a successful verification, set $\zeta=\zeta^{\prime}$ and $\overrightarrow{\mathcal{C}}=\overrightarrow{\mathcal{C}^{\prime}}$, compute witnesses $w_{j}^{i} \leftarrow$ CreateWitness $\left(\mathrm{SP}_{1}, \phi_{i}(x), \hat{\phi}_{i}(x), j\right)$ for $j \in[1, n]$ and $w_{i}^{\mathcal{C}} \leftarrow$ CreateWitness $\left(\mathrm{SP}_{2}, h_{\mathcal{C}}(x), \widehat{h}_{\mathcal{C}}(x), i\right)$.

Send a message (echo, $\zeta^{\prime}$ ) to all parties.

- On receiving (echo, $\left.\zeta^{\prime}\right)$ from at least $(n-t)$ parties:

- For $\zeta^{\prime}=\zeta$, send (ready, $\zeta^{\prime}$, share, $\left.\phi_{i}(j), \hat{\phi}_{i}(j), w_{j}^{i}, \mathcal{C}_{i}, \widehat{h}_{\mathcal{C}}(i), w_{i}^{\mathcal{C}}\right)$ to every party $P_{j}$;

- For $\zeta^{\prime} \neq \zeta$, discard $\left\langle\overrightarrow{\mathcal{C}}, \overrightarrow{\mathcal{W}}_{i}, \phi_{i}(x), \hat{\phi}_{i}(x)\right\rangle$, set $\zeta=\zeta^{\prime}$, and send (ready, $\zeta^{\prime}$, no-share) to all parties.

- If a ready message has not been sent, then on receiving (ready, $\left.\zeta^{\prime}, *\right)$ messages from $(t+1)$ parties:

- For $\zeta^{\prime}=\zeta$, send (ready, $\zeta^{\prime}$, share, $\left.\phi_{i}(j), \hat{\phi}_{i}(j), w_{j}^{i}, \mathcal{C}_{i}, \widehat{h}_{\mathcal{C}}(i), w_{i}^{\mathcal{C}}\right)$ to every party $P_{j}$;

- For $\zeta^{\prime} \neq \zeta$, discard $\left\langle\overrightarrow{\mathcal{C}}, \overrightarrow{\mathcal{W}}_{i}, \phi_{i}(x), \hat{\phi}_{i}(x)\right\rangle$, set $\zeta=\zeta^{\prime}$, and send (ready, $\zeta^{\prime}$, no-share) to all parties.

- On receiving (ready, $\left.\zeta^{\prime}, *\right)$ messages from at least $(n-t)$ parties such that at least $(t+1)$ of those messages contain $\left\langle\right.$ share, $\left.\phi_{j}(i), \hat{\phi}_{j}(i), w_{i}^{j}, \mathcal{C}_{j}, \hat{h}_{\mathcal{C}}(j), w_{\mathcal{C}}^{j}\right\rangle$ successfully verified using VerifyE$\operatorname{val}\left(\mathrm{SP}_{1}, \mathcal{C}_{j}, i, \phi_{j}(i), \hat{\phi}_{j}(i), w_{i}^{j}\right)$ and VerifyEval $\left(\mathrm{SP}_{2}, \zeta^{\prime}, j, H\left(\mathcal{C}_{j}\right), \hat{h}_{\mathcal{C}}(j), w_{\mathcal{C}}^{j}\right)$, interpolate

- shares $\phi_{0}(i)$ and $\hat{\phi}_{0}(i)$ from respectively $(t+1) \phi_{j}(i)$ values and $(t+1) \hat{\phi}_{j}(i)$ values, and

- commitment $\mathcal{C}_{0}$ and witness $w_{i}^{0}$ from respectively $(t+1) \mathcal{C}_{j}$ values and $(t+1) w_{i}^{j}$ values.

Complete the Sh phase with $\left(\zeta=\zeta^{\prime}, \mathcal{C}_{0}, \phi_{0}(i), \hat{\phi}_{0}(i), w_{i}^{0}\right)$ as output.

\section{Every party $P_{i}$}

\section{Rec Phase}

- Send a message (rec-share, $\left.\phi_{0}(i), \hat{\phi}_{0}(i), w_{i}^{0}\right)$ to every party $P_{j}$.

- On receiving $t+1$ rec-share messages verified using $\operatorname{Verify} \operatorname{Eval}\left(\mathrm{SP}_{1}, \mathcal{C}_{0}, \phi_{0}(j), \hat{\phi}_{0}(j), w_{j}^{0}\right)$, interpolate the shares $\phi_{0}(j)$ to compute the secret $s$.

Figure 2: Protocol eAVSS-SC for Asynchronous VSS with Stronger Commitment

\subsection{Analysis}

Theorem 4.1. Given a PolyCommit scheme that satisfies Definition 2.4, eAVSS-SC is an asynchronous VSS protocol that satisfies Definition 2.5 with the strong commitment property. 
Proof Outline. We have to prove that protocol eAVSS satisfies the asynchronous VSS properties in Definition 2.5 along with the strong commitment property. Our analysis is based on the following two claims and the properties of the PolyCommit scheme. We present our proof sketch here, while the complete proof appears in Appendix B.

Claim 4.2. If some honest party agrees on $\zeta$, then every honest party will eventually agree on $\zeta$.

Claim 4.3. All honest servers complete the Sh phase with the same PolyCommit commitment $\mathcal{C}_{0}$.

Proof. Assume two honest parties terminate with $\mathcal{C}_{0}{ }^{\prime}$ and $\mathcal{C}_{0}{ }^{\prime \prime}$ such that $\mathcal{C}_{0}{ }^{\prime} \neq \mathcal{C}_{0}{ }^{\prime \prime}$. From Claim 4.2, we know that all honest parties agree on the same $\zeta$. As $\zeta$ commits to $h_{\mathcal{C}}(x)$, an $n$-degree polynomial interpolated by hashing $n+1$ elements of $\overrightarrow{\mathcal{C}}$, the adversary has to break the evaluation binding property of the polynomial commitment or the collision resistance property of hash function to obtain two different $\mathcal{C}_{0}$ values that culminate the same $\zeta$. This is not possible in PPT and there is a contradiction. Therefore, we prove that all honest servers complete the Sh phase with the same $\mathcal{C}_{0}$.

Liveness is apparent from the protocol flow and correctness of the PolyCommit scheme. Agreement in the Sh phase follows from claims 4.2 and 3.3, while agreement in reconstruction follows from agreement during the Sh phase. Correctness follows directly from correctness of the PolyCommit scheme and collision-resistance of the hash function. Strong Commitment follows from agreement of eAVSS-SC and Claim 3.3. Secrecy follows from the hiding property of PolyCommit.

\subsection{Lower Bounds}

We observe that the $\Omega\left(n^{2}\right)$ message complexity of our eAVSS and eAVSS-SC protocols as well as the AVSS protocol is optimal. ${ }^{3}$ This can be proved in two steps: first, it is known that a VSS protocol is sufficient to implement reliable broadcast [24]; next, extending a result by Dolev and Reischuk [19] for Byzantine agreement to reliable broadcast. The latter proves that if a reliable broadcast protocol terminates, the number of messages exchanged by honest parties is lower bounded by $\max \left\{(n-t),(1+t / 2)^{2}\right\}$ in presence of a commitment scheme. The above two claims show that the message complexity of asynchronous VSS is lower-bounded by $\Omega\left(n^{2}\right)$ for optimal resiliency condition $n=3 t+1$ and $t>2$. We thoroughly prove this result in Appendix $\mathrm{C}$.

Note that when the shared secret is of size $\kappa$ (the computational security parameter), the lower bound of $\Omega\left(n^{2}\right)$ message complexity intuitively transfers to a lower bound of $\Omega\left(\kappa n^{2}\right)$ on the asynchronous VSS communication complexity. Nevertheless, proving this thoroughly presents an interesting challenge. If proven, it will show that our eAVSS and eAVSS-SC protocols are not only optimal in terms of message complexity but also in terms of communication complexity.

\section{Applications}

Our eAVSS and eAVSS-SC schemes have direct implications to all asynchronous VSS applications. We briefly discuss some important applications here.

Using our eAVSS-SC protocol in proactive VSS [12] reduces its communication complexity by a linear factor to $O\left(\kappa n^{3}\right)$. The same reduction also applies to distributed key generation required for threshold cryptography, and its group and threshold modification primitives [22]. Using our eAVSS protocol in the asynchronous Byzantine agreement (ABA) framework of Canetti and Rabin [13,15],

\footnotetext{
${ }^{3}$ With the stronger cryptographic assumptions such as PKI or ZK proofs more efficient schemes can be possible; however, we only assume commitment schemes here.
} 
it is possible to obtain the first $O\left(\kappa n^{3}\right)$ communication complexity ABA protocol, which is secure against the adaptive adversary in the standard model without the random oracle assumption (see [12, Sec. 3.5] for details).

Finally, the commitment methodology used here may also find applications in some other bivariate polynomial-based protocols; however, one has to be careful as it is not a full-fledged bivariate polynomial commitment scheme.

\section{References}

[1] I. Abraham, D. Dolev, and J. Y. Halpern. An Almost-surely Terminating Polynomial Protocol for Asynchronous Byzantine Agreement with Optimal Resilience. In ACM PODC'08, pages 405-414, 2008.

[2] M. H. Au, Q. Wu, W. Susilo, and Y. Mu. Compact E-Cash from Bounded Accumulator. In Proceedings of CT-RSA'0\%, pages 178-195, 2007.

[3] M.H. Au, W. Susilo, and Y. Mu. Practical anonymous divisible e-cash from bounded accumulators. In Proceedings of FC'08, pages 287-301, 2008.

[4] M. Backes and C. Cachin. Reliable broadcast in a computational hybrid model with byzantine faults, crashes, and recoveries. In Intl. Conference on Dependable Systems and Networks-DSN-2003, 2003.

[5] M. Backes, A. Kate, and A. Patra. Computational Verifiable Secret Sharing Revisited. In Advances in Cryptology-ASIACRYPT, pages 590-609, 2011.

[6] M. Ben-Or, R. Canetti, and O. Goldreich. Asynchronous Secure Computation. In ACM STOC'93, pages 52-61, 1993.

[7] G. R. Blakley. Safeguarding Cryptographic Keys. In the National Computer Conference, pages 313-317, 1979.

[8] D. Boneh and X. Boyen. Efficient Selective-ID Secure Identity-Based Encryption Without Random Oracles. In Proceedings of EUROCRYPT'04, pages 223-238, 2004.

[9] D. Boneh and X. Boyen. Short Signatures Without Random Oracles. In Proceedings of EUROCRYPT'04, volume 3027 of LNCS, pages 56-73. Springer, 2004.

[10] D. Boneh, X. Boyen, and E.J. Goh. Hierarchical identity based encryption with constant size ciphertext. In Eurocrypt, volume 3494, pages 440-456. Springer, 2005.

[11] G. Bracha. An Asynchronous [(n-1)/3]-Resilient Consensus Protocol. In PODC'84, pages 154-162, 1984.

[12] C. Cachin, K. Kursawe, A.Lysyanskaya, and R. Strobl. Asynchronous Verifiable Secret Sharing and Proactive Cryptosystems. In $A C M C C S^{\prime} 02$, pages 88-97, 2002.

[13] R. Canetti. Studies in Secure Multiparty Computation and Applications. PhD thesis, The Weizmann Institute of Science, 1996.

[14] R. Canetti, R. Gennaro, S. Jarecki, H. Krawczyk, and T. Rabin. Adaptive Security for Threshold Cryptosystems. In Advances in Cryptology-CRYPTO'99, pages 98-115, 1999.

[15] R. Canetti and T. Rabin. Fast Asynchronous Byzantine Agreement with Optimal Resilience. In ACM STOC'93, pages 42-51, 1993.

[16] J.H. Cheon. Security analysis of the strong Diffie-Hellman problem. In Proceedings of EUROCRYPT'06, volume 4004 of LNCS, pages 1-13. Springer, 2006.

[17] B. Chor, S. Goldwasser, S. Micali, and B. Awerbuch. Verifiable Secret Sharing and Achieving Simultaneity in the Presence of Faults. In IEEE FOCS'85, pages 383-395, 1985. 
[18] R. Cramer, I. Damgård, and S. Dziembowski. On the complexity of verifiable secret sharing and multiparty computation. In $S T O C$, pages 325-334, 2000.

[19] D. Dolev and R. Reischuk. Bounds on information exchange for byzantine agreement. J. ACM, 32(1):191-204, 1985.

[20] P. Feldman. A Practical Scheme for Non-interactive Verifiable Secret Sharing. In IEEE FOCS'87, pages $427-437,1987$.

[21] A. Herzberg, S. Jarecki, H. Krawczyk, and M. Yung. Proactive Secret Sharing Or: How to Cope With Perpetual Leakage. In Proceedings of CRYPTO'95, volume 963 of LNCS, pages 339-352, 1995.

[22] A. Kate and I. Goldberg. Distributed Key Generation for the Internet. In 29th IEEE International Conference on Distributed Computing Systems (ICDCS), pages 119-128, 2009.

[23] A. Kate, G. M. Zaverucha, and I. Goldberg. Constant-Size Commitments to Polynomials and Their Applications. In Proceedings of ASIACRYPT'10, pages 177-194, 2010.

[24] J. Katz, C. Koo, and R. Kumaresan. Improving the round complexity of vss in point-to-point networks. In ICALP(2)'08, pages 499-510, 2008.

[25] S. Mitsunari, R. Sakai, and M. Kasahara. A New Traitor Tracing. IEICE Transactions on Fundamentals of Electronics, Communications and Computer Sciences, E85-A(2):481-484, 2002.

[26] L. Nguyen. Accumulators from bilinear pairings and applications. In Proceedings of CT-RSA, volume 3376 of $L N C S$, pages 275-292, 2005.

[27] A. Patra, A. Choudhary, and C. Pandu Rangan. Efficient Asynchronous Byzantine Agreement with Optimal Resilience. In ACM PODC'09, pages 92-101, 2009.

[28] T. P. Pedersen. Non-Interactive and Information-Theoretic Secure Verifiable Secret Sharing. In Proceedings of CRYPTO'91, pages 129-140. Springer, 1991.

[29] T.P. Pedersen. A Threshold Cryptosystem without a Trusted Party. In Proceedings of EUROCRYPT'91, pages 522-526. Springer, 1991.

[30] D. A. Schultz, B. Liskov, and M. Liskov. MPSS: Mobile Proactive Secret Sharing. ACM Trans. Inf. Syst. Secur., 13(4):34, 2010.

[31] A. Shamir. How to Share a Secret. Commun. ACM, 22(11):612-613, 1979.

[32] L. Zhou, F. B. Schneider, and R. van Renesse. APSS: Proactive Secret Sharing in Asynchronous Systems. ACM Trans. Inf. Syst. Secur. (TISSec), 8(3):259-286, 2005. 


\section{A Protocol PolyCommit ${ }_{\text {Ped }}$ : Constant-size Commitments to Uni- variate Polynomials}

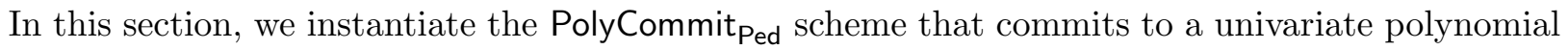
using a single group element.

\section{A.1 Cryptographic Assumptions}

We first discuss the cryptographic assumptions used in the PolyCommit constructions [23].

Definition A.1. Discrete logarithm (DLog) Assumption. Given a generator $g$ of $\mathbb{G}^{*}$, where $\mathbb{G}^{*}=\mathbb{G}$ or $\mathbb{G}_{T}$, and $a \in_{R} \mathbb{Z}_{p}^{*}$, for every adversary $\mathcal{A}_{D L o g}, \operatorname{Pr}\left[\mathcal{A}_{D L o g}\left(g, g^{a}\right)=a\right]=\epsilon(\kappa)$.

Mitsunari, Sakai and Kasahara [25] introduced the weak Diffie-Hellman assumption, which was renamed the $t$-DHI assumption by Boneh and Boyen [8] as this assumption is stronger than the Diffie-Hellman assumption, especially for large values of $t$.

The $t$-DHI problem is, on input $\left\langle g, g^{\alpha}, g^{\alpha^{2}}, \ldots, g^{\alpha^{t}}\right\rangle \in \mathbb{G}^{t+1}$ to output $g^{1 / \alpha}$, or equivalently (see [10]), $g^{\alpha^{t+1}}$. Kate et al. [23] used a generalization of the $t$-DHI assumption, where $\mathcal{A}$ has to output a pair $\left\langle\phi(x), g^{\phi(\alpha)}\right\rangle \in \mathbb{Z}_{p}[x] \times \mathbb{G}$ such that $2^{\kappa}>\operatorname{deg}(\phi)>t$. They named this assumption as the $t$-polynomial Diffie-Hellman (t-polyDH) assumption.

Definition A.2. $t$-polynomial Diffie-Hellman (t-polyDH) Assumption. Let $\alpha \in_{R} \mathbb{Z}_{p}^{*}$. Given as input a $(t+1)$-tuple $\left\langle g, g^{\alpha}, g^{\alpha^{2}}, \ldots, g^{\alpha^{t}}\right\rangle \in \mathbb{G}^{t+1}$, for every adversary $\mathcal{A}_{t-p o l y D H}$, the probability $\operatorname{Pr}\left[\mathcal{A}_{t-p o l y D H}\left(g, g^{\alpha}, g^{\alpha^{2}}, \ldots, g^{\alpha^{t}}\right)=\left\langle\phi(x), g^{\phi(\alpha)}\right\rangle\right]=\epsilon(\kappa)$ for any freely chosen $\phi(x) \in \mathbb{Z}_{p}[x]$ such that $2^{\kappa}>\operatorname{deg}(\phi)>t$.

This assumption was implicitly made by $[2,3]$ to support their claim that the accumulator of $[26]$ is bounded. The $\operatorname{deg}(\phi)$ is bounded by $2^{\kappa}$ as evaluations can be found for polynomials with higher degrees in PPT using number theoretic techniques (e.g., for $\phi(x)=x^{p-1}, g^{\phi(\alpha)}=g$ for any $\left.\alpha \in \mathbb{Z}_{p}^{*}\right)$. In practice, $\operatorname{deg}(\phi) \ll 2^{\kappa}$.

Boneh and Boyen [9] defined the $t$-strong Diffie-Hellman assumption that is related to but stronger than the $t$-DHI assumption and has exponentially many non-trivially different solutions, all of which are acceptable.

Definition A.3. $t$-Strong Diffie-Hellman ( $t$-SDH) Assumption. Let $\alpha \in_{R} \mathbb{Z}_{p}^{*}$. Given as input a $(t+1)$-tuple $\left\langle g, g^{\alpha}, g^{\alpha^{2}}, \ldots, g^{\alpha^{t}}\right\rangle \in \mathbb{G}^{t+1}$, for every adversary $\mathcal{A}_{t-S D H}$, the probability $\operatorname{Pr}\left[\mathcal{A}_{t-S D H}\left(g, g^{\alpha}, g^{\alpha^{2}}, \ldots, g^{\alpha^{t}}\right)=\left\langle c, g^{\frac{1}{\alpha+c}}\right\rangle\right]=\epsilon(\kappa)$ for any value of $c \in \mathbb{Z}_{p} \backslash\{-\alpha\}$.

See Cheon [16] and [23] for security analyses of the above assumptions.

\section{A.2 Construction}

PolyCommit $_{\text {Ped }}$ is based on the algebraic property of polynomials $\phi(x) \in \mathbb{F}_{p}[x]:(x-i)$ perfectly divides the polynomial $\phi(x)-\phi(i)$ for $i \in \mathbb{F}_{p}$. Further, it uses an additional random polynomial $\hat{\phi}(x)$ to achieve unconditional hiding.

Setup $\left(1^{\kappa}, t\right)$ computes two groups $\mathbb{G}$ and $\mathbb{G}_{T}$ of prime order $p$ (providing $\kappa$-bit security) such that there exists a symmetric bilinear pairing $e: \mathbb{G} \times \mathbb{G} \rightarrow \mathbb{G}_{T}$ and for which the $t$-SDH assumption holds. We denote the generated bilinear pairing group as $\mathcal{G}=\left\langle e, \mathbb{G}, \mathbb{G}_{t}\right\rangle$. Choose two generators $g, h \in_{R} \mathbb{G}$. Let $\alpha \in_{R} \mathbb{F}_{p}^{*}$ be SK, generated by a (possibly distributed) trusted 
authority. Setup also generates a $(2 t+2)$-tuple $\left\langle g, g^{\alpha}, \ldots, g^{\alpha^{t}}, h, h^{\alpha}, \ldots, h^{\alpha^{t}}\right\rangle \in \mathbb{G}^{2 t+2}$ and outputs SP $=\left\langle\mathcal{G}, g, g^{\alpha}, \ldots, g^{\alpha^{t}}, h, h^{\alpha}, \ldots, h^{\alpha^{t}}\right\rangle$. Note that SK is not required by the other algorithms of the commitment scheme, and it can be discarded by the authority if $t$ is fixed.

$\operatorname{Commit}(\mathbf{S P}, \phi(x))$ chooses $\hat{\phi}(x) \in \in_{R} \mathbb{F}_{p}[x]$ of degree $t$ and computes commitment $\mathcal{C}=g^{\phi(\alpha)} h^{\hat{\phi}(\alpha)} \in$ $\mathbb{G}$ for the polynomial $\phi(x) \in \mathbb{F}_{p}[X]$ of degree $t$ or less. For $\phi(x)=\sum_{j=0}^{\operatorname{deg}(\phi)} \phi_{j} x^{j}$ and $\hat{\phi}(x)=$ $\sum_{j=0}^{\operatorname{deg}(\hat{\phi})} \hat{\phi}_{j} x^{j}$, it outputs $\mathcal{C}=\prod_{j=0}^{\operatorname{deg}(\phi)}\left(g^{\alpha^{j}}\right)^{\phi_{j}} \prod_{j=0}^{\operatorname{deg}(\hat{\phi})}\left(h^{\alpha^{j}}\right)^{\hat{\phi}_{j}}$ as the commitment to $\phi(x)$.

$\operatorname{Open}(\mathbf{S P}, \mathcal{C}, \phi(x), \hat{\phi}(x))$ outputs the committed polynomials $\phi(x)$ and $\hat{\phi}(x)$.

$\operatorname{VerifyPoly}(\mathbf{S P}, \mathcal{C}, \phi(x), \hat{\phi}(x))$ verifies that $\mathcal{C} \stackrel{?}{=} g^{\phi(\alpha)} h^{\hat{\phi}(\alpha)}$. If $\mathcal{C}=\prod_{j=0}^{\operatorname{deg}(\phi)}\left(g^{\alpha^{j}}\right)^{\phi_{j}} \prod_{j=0}^{\operatorname{deg}(\hat{\phi})}\left(h^{\alpha^{j}}\right)^{\hat{\phi}_{j}}$ for $\phi(x)=\sum_{j=0}^{\operatorname{deg}(\phi)} \phi_{j} x^{j}$ and $\hat{\phi}(x)=\sum_{j=0}^{\operatorname{deg}(\hat{\phi})} \hat{\phi}_{j} x^{j}$, the algorithm outputs 1 , else it outputs 0. Note that this only works when $\operatorname{both} \operatorname{deg}(\phi)$ and $\operatorname{deg}(\hat{\phi}) \leq t$.

CreateWitness $(\mathbf{S P}, \phi(x), \hat{\phi}(x), i)$ computes $\psi_{i}(x)=\frac{\phi(x)-\phi(i)}{(x-i)}$ and $\hat{\psi}_{i}(x)=\frac{\hat{\phi}(x)-\hat{\phi}(i)}{(x-i)}$, and outputs $\left\langle i, \phi(i), \hat{\phi}(i), w_{i}\right\rangle$. Here, the witness $w_{i}=g^{\psi_{i}(\alpha)} h^{\hat{\psi}_{i}(\alpha)}$.

VerifyEval(SP, $\left.\mathcal{C}, i, \phi(i), \hat{\phi}(i), w_{i}\right)$ verifies that $\phi(i)$ is the evaluation at the index $i$ of the polynomial committed to by $\mathcal{C}$. If $e(\mathcal{C}, g) \stackrel{?}{=} e\left(w_{i}, g^{\alpha} / g^{i}\right) e\left(g^{\phi(i)} h^{\hat{\phi}(i)}, g\right)$, the algorithm outputs 1 , else it outputs 0 .

Suppose $h=g^{\lambda}$ for some unknown $\lambda$. Then VerifyEval is correct because

$$
\begin{aligned}
e\left(w_{i}, g^{\alpha} / g^{i}\right) e\left(g^{\phi(i)} h^{\hat{\phi}(i)}, g\right)= & e\left(g^{\psi_{i}(\alpha)+\lambda \hat{\psi}_{i}(\alpha)}, g^{(\alpha-i)}\right) e(g, g)^{\phi(i)+\lambda \hat{\phi}(i)} \\
& =e(g, g)^{\left(\psi_{i}(\alpha)(\alpha-i)+\phi(i)\right)+\lambda\left(\hat{\psi}_{i}(\alpha)(\alpha-i)+\hat{\phi}(i)\right)} \\
& =e(g, g)^{\phi(\alpha)+\lambda \hat{\phi}(\alpha)} \text { as } \phi(x)=\psi_{i}(x)(x-i)+\phi(i) \\
& \quad \text { and } \hat{\phi}(x)=\hat{\psi}_{i}(x)(x-i)+\hat{\phi}(i) \\
= & e\left(g^{\phi(\alpha)} h^{\hat{\phi}(\alpha)}, g\right)=e(\mathcal{C}, g)
\end{aligned}
$$

The hiding property of PolyCommit Ped $_{\text {is }}$ unconditional. The polynomial binding property is based on the DLog assumption, while the evaluation binding property is based on the $t$-SDH assumption. The strong correctness property follows from the $t$-polyDH assumption.

\section{B Complete Proof for Theorem 4.1}

To prove the theorem, we show that protocol eAVSS-SC satisfies liveness, agreement, correctness, strong commitment, secrecy properties of asynchronous VSS according to Definition 2.5. Our analysis is based on the properties of the polynomial commitment scheme.

We start our discussion with the following two claims.

Claim B.1. If some honest party agrees on $\zeta$, then every honest party will eventually agree on $\zeta$.

This follows from directly from Claim 3.2.

Claim B.2. All honest servers complete the Sh phase with the same PolyCommit commitment $\mathcal{C}_{0}$. 
Proof. Assume two honest parties terminate with $\mathcal{C}_{0}{ }^{\prime}$ and $\mathcal{C}_{0}{ }^{\prime \prime}$ such that $\mathcal{C}_{0}{ }^{\prime} \neq \mathcal{C}_{0}{ }^{\prime \prime}$. From Claim B.1, we know that all honest parties agree on the same $\zeta$. As $\zeta$ commits to $h_{\mathcal{C}}(x)$, an $n$-degree polynomial interpolated by hashing $n+1$ elements of $\overrightarrow{\mathcal{C}}$, the adversary has to break the evaluation binding property of the polynomial commitment or the collision resistance property of hash function to obtain two different $\mathcal{C}_{0}$ values that culminate the same $\zeta$. This is not possible in PPT and there is a contradiction. Therefore, we prove that all honest servers complete the Sh phase with the same $\mathcal{C}_{0}$.

Liveness. Given dealer $P_{d}$ is honest and shares a secret $s$, verification of send message by each

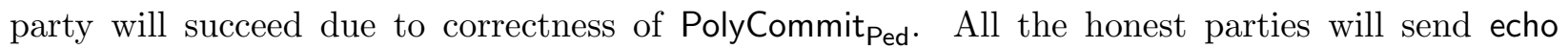
messages for the same $\zeta$ and consequently will send ready messages for the same $\zeta$ with the share tag and the shares. Therefore, at least $n-t$ ready messages will be communicated with share tag, and all parties will obtain their shares of the secret and end with the same commitment $\mathcal{C}_{0}$. During the Rec phase, all $n-t$ honest parties will send their shares consistent with $\mathcal{C}_{0}$ and output the dealer's secret $s$.

Agreement. We first show that if any honest party completes the Sh phase, then every honest party will eventually complete the sharing phase. Claim B.2 proves that all honest servers complete the $\mathrm{Sh}$ phase with the same commitment $\mathcal{C}_{0}$. On the similar lines, using Claim B.1 and strong correctness of PolyCommit ${ }_{\text {Ped }}$, we show that every honest party $P_{i}$ also completes the Sh phase with $\left\langle s_{i}, \hat{s}_{i}, w_{i}^{0}\right\rangle$ consistent with $\mathcal{C}_{0}$ for the index $i$; i.e., $\mathcal{C}_{0}$ commits to a $t$-degree polynomial $\phi_{0}(x)$ such that $s_{i}=\phi_{0}(i)$. This proves the agreement in the Sh phase. As all honest parties terminate with a valid share of the secret $s_{i}$, commitments $\mathcal{C}_{0}$ and witnesses, all honest servers complete the Rec phase with the same output $\phi_{0}(0)$.

Correctness. Assume that the honest dealer has shared a secret $s$ using $\phi(x, y)$, and has remained honest throughout the execution of the Sh protocol. Let $\zeta$ and $\overrightarrow{\mathcal{C}}$ be the commitment to $\phi(x)$ spread by the dealer. Given correctness of the polynomial commitment scheme and the collision-resistance property of the hash function, all honest parties will hold correct shares of the secret $s$ that is consistent with $\zeta$ and $\overrightarrow{\mathcal{C}}$

Strong Commitment. In the agreement property proof, we show that all honest parties receive their shares in the Sh phase, therefore, we only need to prove the correct property. Assume that two honest parties reconstruct values $s^{\prime}$ and $s^{\prime \prime}$ such that $s^{\prime} \neq s^{\prime \prime}$. This implies that they received distinct subsets $\mathcal{S}^{\prime}=\left\{\left(s_{(j)}, \hat{s}_{(j)}, w_{0}{ }^{(j)}\right)\right\}$ and $\mathcal{S}^{\prime \prime}=\left\{\left(s_{(j)}, \hat{s}_{(j)}, w_{0}{ }^{(j)}\right)\right\}$ of the size $(t+1)$ such that each of those satisfies VerifyEval $\left(\mathrm{SP}_{1}, \mathcal{C}_{0}, l, s_{j}, \hat{s}_{j}, w^{0}{ }_{j}\right)$ with the same $\mathcal{C}_{0}$, which follows from Lemma B.2. A probability of that is negligible according to the evaluation binding property of PolyCommit Ped $_{\text {. }}$ and we obtain the required contradiction.

Secrecy. We show that upon obtaining send messages $\left\langle\left(\text { send, } \zeta, \overrightarrow{\mathcal{C}}, \widehat{h}_{\mathcal{C}}(x), \overrightarrow{\mathcal{W}}_{i}, \phi_{i}(x), \hat{\phi}_{i}(x)\right)\right\rangle_{i \in I}$ and ready messages $\left\langle\left(\operatorname{ready}, \phi_{j}(i), \hat{\phi}_{j}(i), w_{j}^{i}, \mathcal{C}_{j}, \widehat{h}_{\mathcal{C}}(j), w_{\mathcal{C}}^{j}\right)\right\rangle_{i \in I, j \in[1, n]}$, the adversary has no information about the secret, where $I$ is a set of compromised $t$ parties

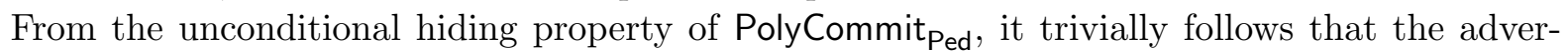
sary obtains no information about $\phi_{i^{\prime}}(j)$ for $i^{\prime} \notin I$ and $j \in[1, n]$ using $t$ tuples of the form $\left\langle\overrightarrow{\mathcal{C}}, \overrightarrow{\mathcal{W}}_{i}, \phi_{i}(x), \hat{\phi}_{i}(x)\right\rangle$. Further, for ready messages, it is easy to observe that the adversary can generate all ready messages it receives using the information provided in the received send messages using the symmetry of polynomials $\phi(x, y), \hat{\phi}(x, y)$ and the witness matrix $\overrightarrow{\mathcal{W}}$. 


\section{Lower Bound on Message Complexity}

We start our discussion by defining a reliable broadcast protocol [11], which is a fundamental synchronization primitive. Here, a distinguished party delivers a message $m$ to $n$ parties in presence of $t$ malicious parties such that if the sender is correct then all honest parties eventually accept $m$, and even if the sender is faulty, all honest parties eventually decide for the same value or do not terminate the protocol at all. In the absence of a proper reliable broadcast definition in [11], we use a definition by Backes and Cachin in [4], which extends Bracha's reliable broadcast work.

Definition C.1. [4] A reliable broadcast protocol in an asynchronous network of $n \geq 3 t+1$ parties with a t-limited Byzantine adversary satisfies the following conditions:

Validity: If an honest dealer broadcasts a message $m$ then all honest parties will deliver this message, provided the adversary delivers all associated messages.

Consistency: If an honest party delivers a message $m$ and another honest party delivers a message $m^{\prime}$, then $m=m^{\prime}$.

Totality: If some honest party delivers a message, then all honest parties deliver a message, provided the adversary delivers all associated messages.

Authenticity: Every honest party delivers at most one message and, if the sender is honest, this message has been broadcast by this sender before.

Lemma C.2. In the asynchronous communication model, an ( $n, t)$-VSS instance implements $(n, t)$ reliable broadcast.

Proof. Without loss of generality, assume that a message $m$ to be broadcast belongs to $\mathbb{F}_{p}$ of the size $\kappa$. To implement reliable broadcast using VSS, the sender, acting as a dealer, appropriately shares the message $m$ with $n$ parties using $(n, t)$-VSS over $\mathbb{F}_{p}$. All honest parties start the Rec phase once they complete their Sh phases successfully, and output the reconstructed value as a broadcast message.

The properties of reliable broadcast (Definition C.1) follow directly from the properties in asynchronous VSS (Definition 2.5), as follows. Validity of reliable broadcast follows directly from liveness and correctness of asynchronous VSS. Consistency of reliable broadcast is equivalent to the commitment property of asynchronous VSS, while totality is exactly the same as agreement of asynchronous VSS. Finally, authenticity of reliable broadcast follows from the fact that in asynchronous VSS, each honest party will output only the value that has been reconstructed in the Rec phase. The second part of the authenticity definition is equivalent to correctness of asynchronous VSS.

Lemma C.3. In the computational setting with commitments, if a reliable broadcast protocol terminates, the total number of messages exchanged by honest parties is lower bounded by max $\{(n-$ $\left.t),(1+t / 2)^{2}\right\}$.

Proof. Dolev and Reischuk [19] showed that a lower bound exists in the total number of messages exchanged among the processors when achieving Byzantine agreement. We use a similar approach to show that reliable broadcast achieves identical lower bounds. For ease of exposition, we do not assume cryptographic commitments initially.

For the set $\mathcal{P}$ of $n$ parties, a phase is a directed graph, where an edge labeled $m$ from $P_{i}$ to $P_{j}$ represents that $P_{i}$ has sent the message $m$ to $P_{j}$. A sequence of such phases in an execution makes up a history for the set $\mathcal{P}$. For each history $H$, there exists an individual subhistory for a party $P_{i}, H_{P_{i}}$. We shall show that there exists a history $H$ in which the honest parties send at least $\max \left\{(n-t),(1+t / 2)^{2}\right\}$ messages. 
Let $H$ be a history in which all the parties are honest and the dealer sends the message $m$. The honest parties cannot reach a conclusion without having received any messages. Therefore, all $n-t$ honest parties must receive at least one message each and at least $n-t$ messages have been sent in $H$.

Now, lets assume that the second term achieves the maximum. Let $\mathcal{C}$ be a set of parties in $\mathcal{P}$ of size $\lfloor 1+t / 2\rfloor$, and let $\mathcal{R}$ be the remaining parties. We shall now show that there exists a history $H^{\prime}$ in which each party in $\mathcal{C}$ is corrupted and honest parties in $\mathcal{R}$ are forced to send at least $\lceil 1+t / 2\rceil$ messages to each party in $\mathcal{C}$.

Let $H^{\prime}$ be the following history: All parties in $\mathcal{R}$ are honest, and the dealer $P_{d}$ correctly sends the message $m$. Each party in $\mathcal{C}$ behaves like an honest party except that it rejects the first $\lceil t / 2\rceil$ messages from the parties in $\mathcal{R}$. If some party receives less than $\lceil t / 2\rceil$ messages, then it ignores all of them. There is no communication between the parties in $\mathcal{C}$. This defines a valid history with $\lfloor 1+t / 2\rfloor$ corrupt parties. In this history, the honest parties must agree on $m$, since the dealer shared this message correctly.

Now, assume that a party $P_{i}$ in $\mathcal{C}$ receives less than $\lceil t / 2\rceil$ messages. We shall show that this assumption leads to a contradiction. Let $R\left(P_{i}\right)$ be the set of parties in $\mathcal{R}$ which had sent messages to $P_{i}$ in $H^{\prime}$. In order to get a contradiction, we now define a new history $H^{\prime \prime}$, where $P_{i}$ is an honest party, and $R\left(P_{i}\right)$ are all corrupted. These parties do not send any message to $P_{i}$, and every party in $\mathcal{C}$ other than $P_{i}$ ignore every message from $P_{i}$.

The corrupted parties in $\mathcal{C}-\left\{P_{i}\right\}$ and $R\left(P_{i}\right)$ behave toward the honest parties in $\mathcal{R}$ in $H^{\prime \prime}$ in exactly the same way as they do in $H^{\prime}$. Since $P_{i}$ in $H^{\prime \prime}$ behaves like an honest party which did not receive the first $\lfloor t / 2\rfloor$ messages, there is no difference in its behavior towards the honest parties of $\mathcal{R}$ in $H^{\prime}$ and $H^{\prime \prime}$. Thus, each honest party $P_{j}$ other than $P_{i}$ observes the same subhistory in $H^{\prime}$ and $H^{\prime \prime}$, i.e. $H_{P_{j}}^{\prime}=H_{P_{j}}^{\prime \prime}$ for all $j \neq i$, and in the end must agree on $m$. But, the honest $P_{i}$ does not receive any message in $H^{\prime \prime}$. Hence, it cannot satisfy totality. This leads to a contradiction, which proves that every party in $\mathcal{C}$ must receive at least $\lceil t / 2\rceil$ messages from the honest parties.

Now, we consider the effect of cryptographic commitments. In case of the honest dealer, $(n-t)$ messages are still necessary as every honest party has to receive some message before concluding anything. A commitment scheme cannot reduce the number of interactions in the latter case either as a commitment does not bind the sender to a single message across multiple receivers; the sender can still send different commitments (and correspondingly different messages) to different honest parties. Therefore, all honest parties have to interact with each other in the exactly same manner resulting in $(1+t / 2)^{2}$ messages. 